\title{
GUIDEseq: a bioconductor package to analyze GUIDE-Seq datasets for CRISPR-Cas nucleases
}

Lihua Julie Zhu' ${ }^{1,2,3^{*}}$, Michael Lawrence ${ }^{4}$, Ankit Gupta ${ }^{1}$, Hervé Pagès ${ }^{5}$, Alper Kucukural ${ }^{3}$, Manuel Garber ${ }^{2,3}$ and Scot A. Wolfe $e^{1,6}$

\begin{abstract}
Background: Genome editing technologies developed around the CRISPR-Cas9 nuclease system have facilitated the investigation of a broad range of biological questions. These nucleases also hold tremendous promise for treating a variety of genetic disorders. In the context of their therapeutic application, it is important to identify the spectrum of genomic sequences that are cleaved by a candidate nuclease when programmed with a particular guide RNA, as well as the cleavage efficiency of these sites. Powerful new experimental approaches, such as GUIDE-seq, facilitate the sensitive, unbiased genome-wide detection of nuclease cleavage sites within the genome. Flexible bioinformatics analysis tools for processing GUIDE-seq data are needed.

Results: Here, we describe an open source, open development software suite, GUIDEseq, for GUIDE-seq data analysis and annotation as a Bioconductor package in R. The GUIDEseq package provides a flexible platform with more than 60 adjustable parameters for the analysis of datasets associated with custom nuclease applications. These parameters allow data analysis to be tailored to different nuclease platforms with different length and complexity in their guide and PAM recognition sequences or their DNA cleavage position. They also enable users to customize sequence aggregation criteria, and vary peak calling thresholds that can influence the number of potential off-target sites recovered. GUIDEseg also annotates potential off-target sites that overlap with genes based on genome annotation information, as these may be the most important off-target sites for further characterization. In addition, GUIDEseq enables the comparison and visualization of off-target site overlap between different datasets for a rapid comparison of different nuclease configurations or experimental conditions. For each identified offtarget, the GUIDEseq package outputs mapped GUIDE-Seq read count as well as cleavage score from a user specified off-target cleavage score prediction algorithm permitting the identification of genomic sequences with unexpected cleavage activity.

Conclusion: The GUIDEseq package enables analysis of GUIDE-data from various nuclease platforms for any species with a defined genomic sequence. This software package has been used successfully to analyze several GUIDE-seq datasets. The software, source code and documentation are freely available at http://www.bioconductor.org/ packages/release/bioc/html/GUIDEseq.html.
\end{abstract}

Keywords: Genome editing, CRISPR, GUIDE-seq, Off-targets analysis, Bioconductor

\footnotetext{
*Correspondence: julie.zhu@umassmed.edu

'Department of Molecular, Cell and Cancer Biology, University of

Massachusetts Medical School, Worcester, MA, USA

${ }^{2}$ Program in Bioinformatics and Integrative Biology, University of

Massachusetts Medical School, Worcester, MA, USA

Full list of author information is available at the end of the article
}

(c) The Author(s). 2017 Open Access This article is distributed under the terms of the Creative Commons Attribution 4.0 International License (http://creativecommons.org/licenses/by/4.0/), which permits unrestricted use, distribution, and reproduction in any medium, provided you give appropriate credit to the original author(s) and the source, provide a link to the Creative Commons license, and indicate if changes were made. The Creative Commons Public Domain Dedication waiver (http://creativecommons.org/publicdomain/zero/1.0/) applies to the data made available in this article, unless otherwise stated. 


\section{Background}

Type II CRISPR/Cas adaptive defense systems employ a single, large multi-subunit endonuclease (Cas9) and a pair of RNAs that as a complex mediate sequence-specific targeted cleavage of foreign DNA [1]. This system has been repurposed into a powerful two-component system (Cas9 \& single guide RNA (sgRNA)) for targeted genome editing $[2,3]$. The Cas9-sgRNA complex is straightforward to target to a desired DNA sequence because sequence-specific recognition is achieved primarily through Watson-Crick pairing of the associated sgRNA. The short Protospacer Adjacent Motif (PAM), which is recognized by the Cas9 protein, is the chief constraint on the target site design density within a genome, although the activity of the Cas9-sgRNA complex is influenced by both target sequence composition and biological features $[4,5]$. Because of its simplicity and efficacy, this technology is revolutionizing experimental approaches in the biological sciences and holds tremendous promise for therapeutic applications [6, 7].

Cleavage of unintended sequences within the genome is one concern associated with the therapeutic application of CRISPR-Cas9 nucleases [8]. S. pyogenes Cas9 (SpCas9)-based nucleases can cleave an imperfect heteroduplex formed between the guide sequence and a DNA sequence containing a functional PAM [9-16], where the number, position and type of base mismatches impact its level of activity [11, 12, 16]. Deep sequencing analysis of potential off-target sites [11-14, 17, 18] from populations of SpCas9-sgRNA treated cells revealed that the majority of mismatched sequences are not appreciably cleaved, but that a subset of these "off-target" sites are functional $[11-14,17,18]$, where up to six mismatches $[19,20]$ or a single base bulge $[15,19,21]$ between the guide and genomic sequence can be tolerated under some conditions. To address this inherent promiscuity, SpCas9 variants with improved precision have been developed [13, 16, 22-28] that can dramatically reduce off-target activity. In addition, other Cas9 [21, 29, 30] and Cpf1 [31, 32] orthologs have precision that is comparable or superior to SpCas9. Despite these advances nuclease precision is still target site dependent. Consequently, for therapeutic applications an unbiased assessment of genome-wide nuclease activity is warranted, since DNA breaks at unintended sites could alter gene expression or gene function through direct mutagenesis or genomic rearrangements [33-37].

A new suite of genome-wide off-target detection methods have been described that can identify genomic sites with moderate to low cleavage activity within a population of nuclease-treated cells [19-21, 33, 38]. One of the most sensitive and straightforward methods to employ is GUIDEseq [19]. This method relies on NHEJ-mediated DNA repair to capture co-introduced blunt-ended double stranded oligonucleotides (dsODNs) at nuclease-induced breakpoints within the genome, thereby tagging these loci for selective amplification and subsequent deep sequencing. GUIDE-seq is quite sensitive, as off-target sites with $>0.1 \%$ indel frequency can be detected [19]. Importantly, the frequency of dsODN insertion appears to be correlated with the frequency of Cas9-induced lesions at each site [19]. GUIDE-seq has been used successfully to evaluate the precision of SpCas9 [19, 39], SpCas9 variants [19, 25, 27, 28] and two Cpf1 orthologs [32].

While the GUIDE-seq method is straightforward to employ, its data processing is complex [19]. GUIDE-seq combines two complementary libraries to define the location of dsODN insertions within the genome. In addition, it uses a non-standard indexing method with a unique molecular index to filter out duplicate sequences that arise during the PCR amplification steps. Currently, only a single bioinformatics application has been released to the community to support the analysis of GUIDE-seq data [40]. Although the existing tool has successfully been used for analyzing GUIDEseq data from SpCas9 [19], the released version is not ideal for nucleases that employ guides of longer length or more complex PAM recognition patterns, where control over the number of mismatches allowed within each element for off-target identification is potentially valuable $[21,29,41,42]$. In addition, the existing tool does not support the comparative analysis of GUIDE-seq studies across related guides or Cas9 variants, which can be useful when evaluating guide/Cas9 variant combinations with the most favorable precision.

In the course of establishing GUIDE-seq in our laboratories [25, 39], we developed an extensively documented Bioconductor package GUIDEseq that provides a flexible tool for the analysis of GUIDE-seq datasets interrogating nuclease specificity. GUIDEseq utilizes a rich parameter set that permits adaptation to the characteristics of alternate nuclease platforms (e.g. variants or orthologs of Cas9 [21, 29, 41] or Cpf1 [42]), such as different length and complexity in their guide and PAM recognition sequences or their DNA cleavage position. Importantly, these parameters facilitate flexible filtering criteria for peak calling and for off-target site assignment, which can be critical for the capture of potential off-target sites depending on the type of nuclease system that is employed. Our software can also annotate offtarget sites to indicate whether they fall within a critical region of the genome, such as the exon of a gene. In addition, our algorithm allows multiple GUIDE-seq datasets from different experiments to be compared to identify cleaved genomic sites that are overlapping or unique for a particular guide RNA/nuclease within a group.

\section{Implementation}

\section{Implementation platform}

GUIDEseq implements a common workflow for GUIDEseq data analysis and annotation as a Bioconductor 
package in R [43, 44]. Developing GUIDEseq as a Bioconductor package allows us to leverage a large number of existing genome analysis [45-49] and visualization [50] tools supported within the Bioconductor project. In addition, the rich annotation data for assembled genomes that are available can be used to associate genomic features with identified off-target sites. Bioconductor is an open source and open development software project (http://www.bioconductor.org), which is updated twice a year, where the current release (Bioconductor 3.5) consists of more than two thousand software and annotation packages. These include many species-specific BSgenome packages for accessing different reference genomic sequences, as well as $O r g D b$ and $T x D b$ annotation packages for accessing annotation information for a variety of species. In addition, utilities are provided within the Bioconductor project to forge customized BSgenome and $T x D b$ packages. These resources provide end-users with a flexible, stable and up-to-date platform for implementing data analysis for a variety of different systems or for customizing the output for a specific system of interest.

\section{Read preprocessing, mapping, filtering, peak calling and off-target identification}

In the GUIDE-seq protocol [19], two different paired-end sequencing libraries are generated from the genomic DNA from each nuclease treatment group. These libraries (forward and reverse) differ in the primers (complementary to one of the two strands of the GUIDE-seq oligonucleotides) that are utilized to amplify genomic regions that are "tagged" by GUIDE-seq oligonucleotide integration. During the construction of these libraries a UMI is incorporated during the distal adaptor ligation, which identifies unique sequencing reads within the paired-end sequencing run. Unique sequencing reads are then aggregated within a defined window and peaks that are potential offtarget sites are identified using data from both libraries based on end-user supplied filtering criteria.

Within our GUIDEseq package, we have integrated powerful sequence analysis algorithms and functionalities from other Bioconductor packages for many stages of the GUIDE-seq data processing. In addition, our GUIDEseq package provides more than 60 adjustable parameters to describe the nuclease sequence preference and allow customized data analysis. For simplicity, the majority of nuclease-specific parameters are preset to correspond to the standard SpCas9 nuclease system, but they can be modified to conform to the characteristics of alternate nuclease platforms (e.g. gRNA sequence and length, and PAM sequence preference and position relative to protospacer). The data analysis parameters permit detailed adjustment of the read filtering criteria, peakcalling parameters (read aggregation window size and coverage threshold), and peak merging criteria. Importantly, extensive documentation is included describing the parameters for customization of this package.

An overview of the GUIDEseq analysis workflow is given in Fig. 1. Species-specific genomic sequence and annotations are loaded using BSgenome, $T x D b$ and orgAnn packages. Preprocessing scripts to extract the UMI sequence, bin sequencing reads associated with different libraries based on the index sequences, remove the constant dsODN sequences, and map the resulting sequencing reads to a desired genome assembly are available at http://mccb.umassmed.edu/GUIDE-seq/. Preprocessing steps are described in detail in the Supplementary Methods section [see Additional file 1]. Within the

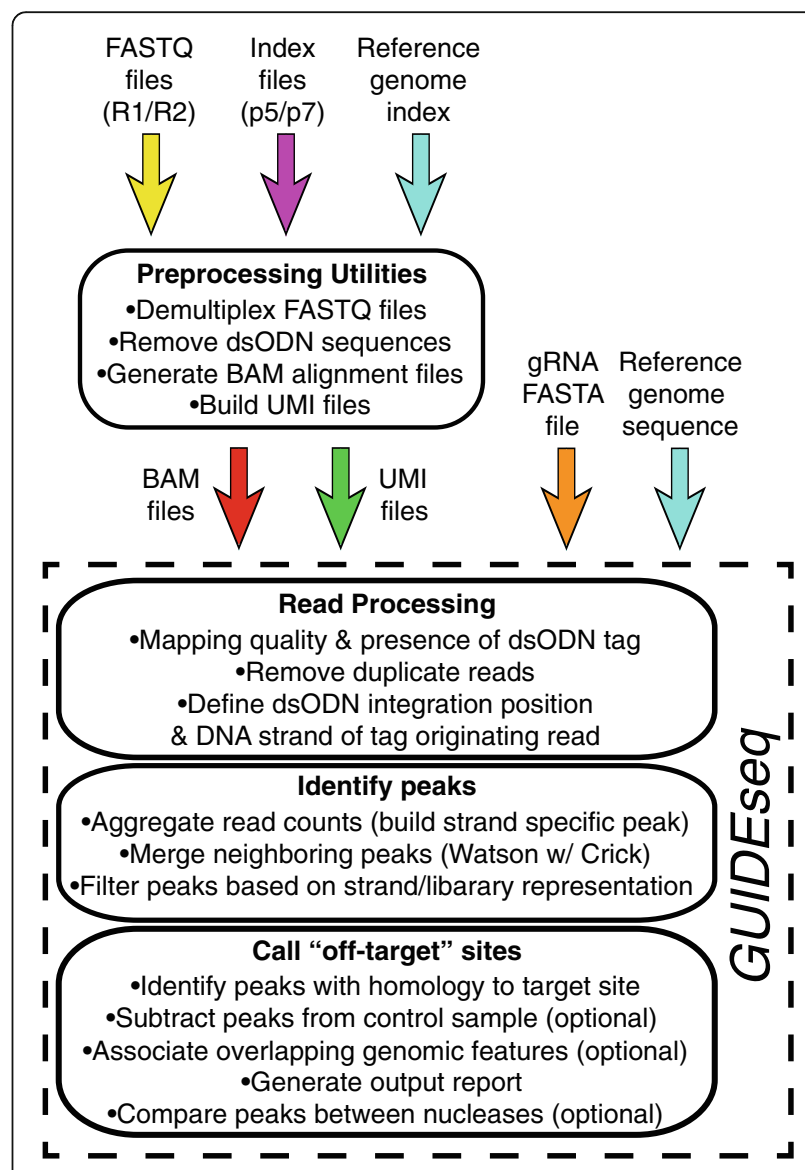

Fig. 1 Overview of GUIDEseq Analysis Workflow. Schematic representation of the GUIDEsea analysis pipeline. Input files required for preprocessing and GUIDEseq package are represented by annotated color arrows. First, Preprocessing Utilities are supplied to demultiplex the Illumina FASTQ files based on the index information and map the sequence files to the reference genome. This generates the experimental input files (BAM and UMI files) needed for the GUIDEsea pipeline, which are supplemented with information on the guide RNA (gRNA) and PAM element by the end-user. Key steps carried out by the algorithms within the GUIDEseq pipeline are indicated under the different headers. Details about the R-based commands and variables used within GUIDEseq are presented in the Use Cases within the main text, and are described in full in the Installation and Usage Section [see Additional file 1] and in the manual pages associated with the program 
GUIDEseq package, read alignments are filtered to remove paired reads that lack a segment of the GUIDE-seq oligonucleotide sequence (reads originating from a dsODN insertion should contain a segment of its sequence; Fig. 2). In addition, paired reads that are too far away from each other, or that are of insufficient length or mapping quality are removed. Because PCR amplification leads to a biased representation of the starting sequence population, paired reads from the same library that share an identical genomic location for the genomic adaptor ligation site, dsODN insertion site, and UMI sequence are collapsed together into a single paired read (Fig. 2). For the purposes of peak calling at the putative nuclease cleavage site, these data are represented as a single genomic position defined by the GUIDE-seq dsODN insertion site with the strand corresponding to Read 2 (Fig. 2). All alignment filtering criteria have a default setting but can be easily adjusted by users.

Unique putative cleavage sites from the forward and reverse libraries are merged and clusters of these sites on the same strand (Watson or Crick) are aggregated over a user-defined sliding window of a specific sequence length (default $=20$ nucleotides, Fig. 3). The height of each strand-specific peak equals the sum of the unique putative cleavage sites within the window, and its position is defined by the center of the 20 base window. Peak calling also filters out clusters with a small number of putative cleavage sites or a high pvalue calculated from a Poisson distribution based on the local background estimate (default to a $5 \mathrm{~kb}$ window). Next, the incorporated ChIPpeakAnno package merges neighboring peaks on the Watson and Crick strands within a defined distance threshold if they have the correct polarity (Crick peaks should precede Watson peaks, Fig. 3) [46, 47]. The height of the merged peaks equals the sum of the heights of the individual peaks, and the location parameter captures the positions of the merged peaks. By default, peaks that lack a Watson/Crick pair and that are only present in one library (forward or reverse) are filtered out [19]. If desired, (nuclease-independent) genomic hotspots for oligonucleotide integration can be removed by comparison against a nuclease-free treatment group [19].

Identified peaks are classified as potential off-target sites based on their sequence homology to the guide sequence and the PAM preference of the Cas9 nuclease (target site) that was employed. GUIDEseq provides multiple parameters to adjust the threshold for the calling of potential offtarget sites within or around peaks passing the filtering criteria to allow adaptation to the type of nuclease (PAM preference) and guide sequence that is employed. Classification of an off-target site is defined by a maximum number of allowed base mismatches to the guide sequence and a separate number of allowed base mismatches to the userdefined PAM, which is implemented using the CRISPRseek package and integrated into the GUIDEseq suite [48, 49]. This separation of the filters for the guide and PAM sequences allows different emphasis to be placed on these elements in the search for potential off-target sequences neighboring GUIDE-seq peaks. GUIDEseq calculates an off-target cleavage prediction score for all identified potential off-target sites using mismatch penalty scoring models generated from an experimental dataset for SpCas9 $[5,12]$ or a user supplied scoring matrix. Based on the available genome annotation, off-target sites in potentially critical regions, such as exons, are flagged. These data for all identified potential off-target sites are output in a tab-delimited format for easy manipulation within spreadsheet or

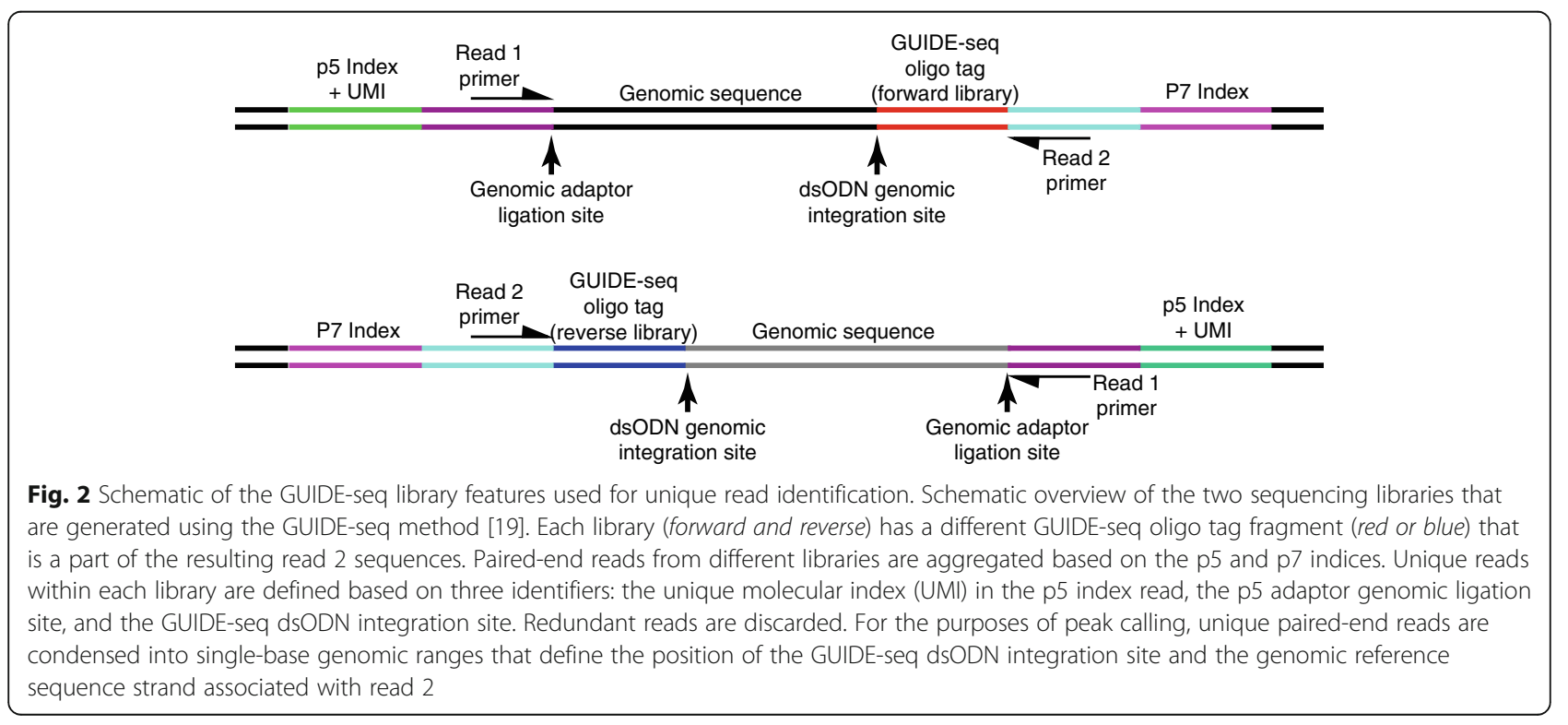




\section{Unique GUIDE-seq read distribution}

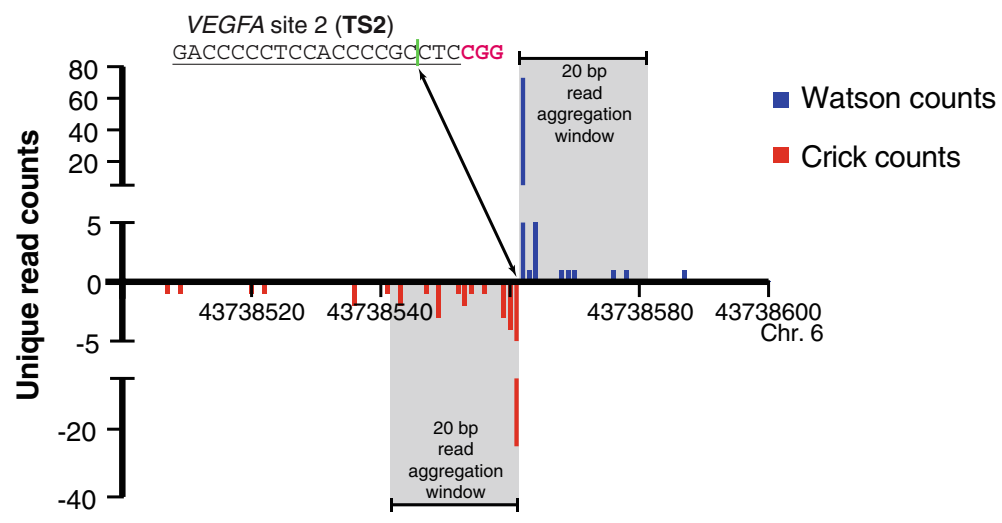

Fig. 3 Unique read aggregation into peaks for the identification of potential nuclease cleavage sites. Strand-specific unique reads defined by the GUIDE-seq dsODN integration site and the read 2 genomic reference sequence strand are aggregated over a user-defined window size (20 base default) to define strand-specific peaks. Windows with a read number greater or equal to a user-defined threshold (default $=5)$ are called peaks. In addition, the signal to noise ratio (SNratio) and a p-value are computed based on the local background window size (defaults $5 \mathrm{~kb}$ and Poisson distribution), which can also be employed as filters if desired. For each integration site, the Crick peak should precede the corresponding Watson peak based on the library construction method [19]. Consequently, this order is required to combine counts from the Watson and Crick peaks over a user-defined window size (40 base default). This aggregate "score" is used to rank peaks. The genomic region surrounding each peak (adjustable variables, default 20 bases on each side) is used to search for sequences with homology to the nuclease sequence preference (based on the input guide sequence ( $g$ RNA.file) and the PAM sequence (PAM), and the allowed mismatches within each element defined by the parameters: max.mismatch, PAM.pattern and allowed.mismatch.PAM. The GUIDE-seq data shown were generated in house for SpCas9 programmed with a sgRNA to recognize VEGFA site 2 (TS2; protospacer underlined, PAM in red) [11], where the most common dsODN integration site falls at the expected cleavage site within this sequence (green line, hg19)

graphing applications. Finally, if a visual comparison of the overlap in identified potential off-target sites between different nucleases or treatment conditions is desired, a Venn diagram of peak overlap in up to five different datasets can be plotted through the integrated limma package [50]. More detailed information on the commands and instructions for running the software are included in the Installation and Usage section [see Additional file 2] and in the associated user guide (https://bioconductor.org/packages/ release/bioc/manuals/GUIDEseq/man/GUIDEseq.pdf and http://bioconductor.org/packages/release/bioc/vignettes/ GUIDEseq/inst/doc/GUIDEseq.pdf).

\section{Results}

\section{Analysis of published GUIDE-seq dataset}

To evaluate the performance of our GUIDEseq analysis package, we analyzed several datasets produced in house and successfully identified the intended target sites and validated GUIDE-seq identified off-targets using deep sequencing of PCR amplicons spanning these genomic loci from nuclease-treated cells [25]. In addition, we analyzed a dataset generously supplied by the Joung laboratory (HEK293 guide 4), and then compared our list of identified off-target sites with their previously published analysis [19]. An example GUIDEseq output file for this dataset is displayed in Additional file 3: Table S1. Each potential off-target site is listed on a separate row ranked based on the peak score (number of unique reads mapped within this region). In addition to the sequence of the potential off-target site, the output includes: the genomic position, its DNA strand, the number of mismatches to the guide sequence and their position and type, and the number of mismatches to the canonical PAM pattern supplied by the user. When gene annotation is supplied for the genome assembly, the transcript (name and entrez-ID) in which the off-target site falls is noted, and whether it occurs within an exon. In addition, for SpCas9, the predicted cleavage score is listed for each site based on the mismatch scoring model generated from the experimental data [5], where a score of 1 indicates predicted activity similar to the target sequence.

When comparing our output to the previously published analysis from the Joung laboratory [19], the number of potential off-target sites and unique reads associated with each peak (their rank order) are very similar (Additional file 4: Table S2). Both outputs are in agreement over the top 90 peaks with only minor differences in the rank order of peaks. The discrepancies between the peak lists are likely due to methodological differences in the sequence filtering, aggregation, and peak-calling criteria that are employed. The unique contributions of our package include its easy adaptability to the analysis of GUIDE-seq datasets from various nuclease platforms, the ability to incorporate annotations of genomic features for identified 
off-target sites and its comparative analysis and visualization features between different GUIDE-seq datasets, which are illustrated in the following use cases.

\section{Use cases}

To simplify the use of the GUIDEseq analysis package, all steps have been integrated into a single workflow function GUIDESeqAnalysis. Once the package is loaded and all of the experiment-specific parameters are set, one line of code (GUIDEseqAnalysis) can perform all the analysis by calling various helper functions. Below are a few examples illustrating how to analyze a GUIDE-seq dataset from three commonly used nucleases, with different PAM orientation, PAM sequence preference, PAM length, gRNA length and reference genome.

\section{Example 1. Analysis of SpCas9 GUIDE-seq data}

Although the analysis workflow function GUIDEseqAnalysis has more than 60 parameters for customized analysis, the majority of these parameters are pre-set for analyzing GUIDE-seq data from the most commonly used nuclease, SpCas9. Consequently when analyzing SpCas9 data only a small number of target-specific inputs are required from users. Detailed description of these parameters and the input files are available at http://bioconductor.org/packages/ release/bioc/manuals/GUIDEseq/man/GUIDEseq.pdf. Information on these parameters can also be accessed from the manual pages by typing help(GUIDEseqAnalysis) in an $\mathrm{R}$ session. Below is an example that defines the required parameters for analysis of SpCas9 data.

First load the required library GUIDEseq and the genome, e.g.,

BSgenome.Hsapiens.UCSC.hg19 for retrieving human genome sequence.

library (GUIDEseq)

library (BSgenome. Hsapiens.UCSC.hg19)

Next create and set the desired working and output directory.

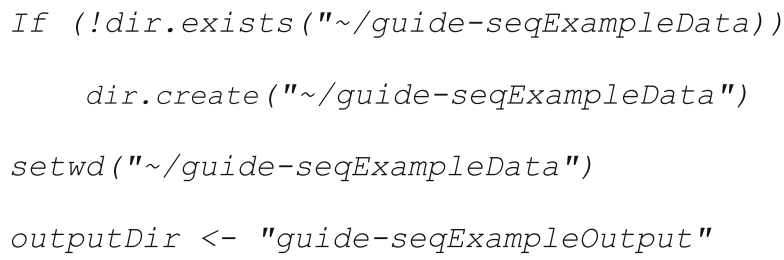

Then set the file paths for target sequence ( $g R N A$.file), sequence alignment (alignment.inputfile) and UMI input files (umi.inputfile). The following code assumes that the input files are located in the current working directory. The gRNA file contains the gRNA sequence in fasta format.

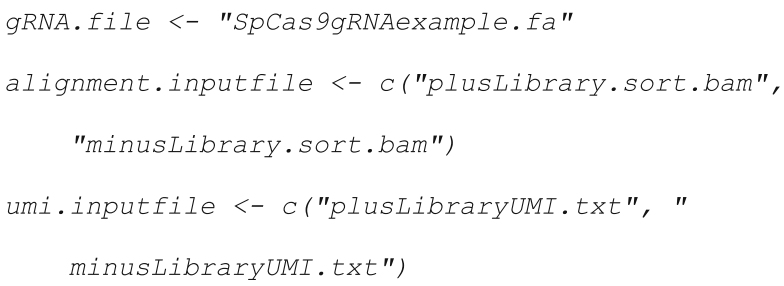

Finally, call the GUIDEseqAnalysis workflow function and save the analysis results in guideSeqResults. The annotated potential off-target sites are output as a tab delimited file (offTargetsInPeakRegions.xls) in the output directory specified by the user.

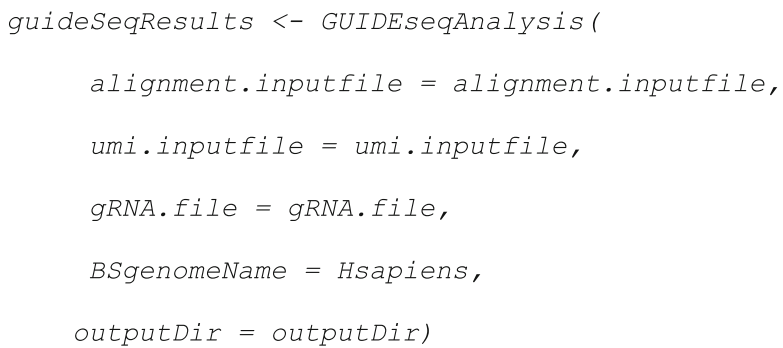

By default, the predicted cleavage score is calculated using the weight matrix and scoring algorithm from the Zhang laboratory [12]. To use the algorithm developed by the Root Laboratory [5] set the scoring.method = "CFDscore". In addition, combineOfftargets (detailed in Example 5 below) can be used to remove off-targets common with a nuclease-free control (e.g. cell type specific double strand break hot spots [19]).

\section{Example 2. Analysis of NmCas9 GUIDE-seq data}

Compared to SpCas9, NmCas9 has a longer gRNA (24 nucleotides), and different PAM sequence preference (NNNNGATT) [41]. Below is an example of the parameters and code needed to analyze GUIDE-seq data from NmCas9. There are only a few additional parameters must be set, i.e., PAM, PAM.size, PAM.pattern, allowed.mismatch.PAM, gRNA.length and weights (to avoid using the SpCas9 default parameters). Currently, there is no positionspecific mismatch penalty matrix available for NmCas9. However, if desired the weight matrix from SpCas9 can be borrowed by simply padding 4 zeros at the beginning of the weight matrix, or an alternate weight matrix can be input (weights). In addition, other parameters that influence the homology search for potential off-target sites within identified peaks should be adjusted. The maximum number of mismatches to the guide (max.mismatch) and PAM (allowed.mismatch.PAM) can be tuned to increase/decrease the specificity/sensitivity of the analysis. In addition, PAM.pattern allows the user to require a specific PAM sequence pattern to be present for additional constraint on the recovered sequences if desired. In the example below potential 
off-target sites are allowed ten mismatches within the guide sequence (24 nucleotides in length), three mismatches within the PAM (8 nucleotides in length), but the PAM is required to have a $\mathrm{G}$ at the fifth position.

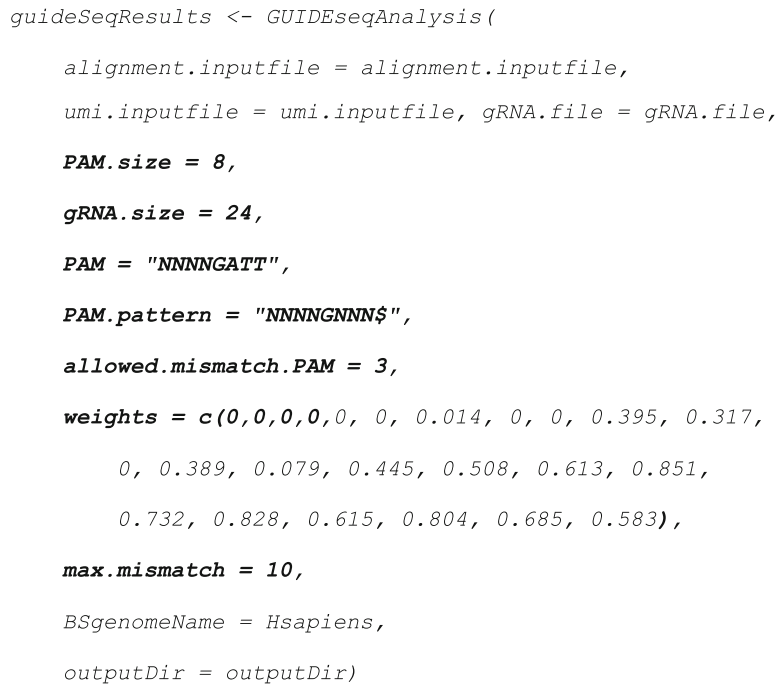

\section{Example 3. Analysis of Cpf1 GUIDE-seq data}

AsCpf1 is an RNA-guided nuclease recognizing a T-rich PAM, TTTN, on the 5 ' side of the protospacer [42], unlike SpCas9, which recognizes an NGG PAM on the 3' side of the protospacer. Below is an example of the parameters and code to analyze a GUIDE-seq dataset for AsCpf1. In addition to the parameters discussed for $\mathrm{NmCas} 9$, there is one more parameter to be changed, i.e., PAM.location, which sets the PAM to the 5' side of the protospacer.

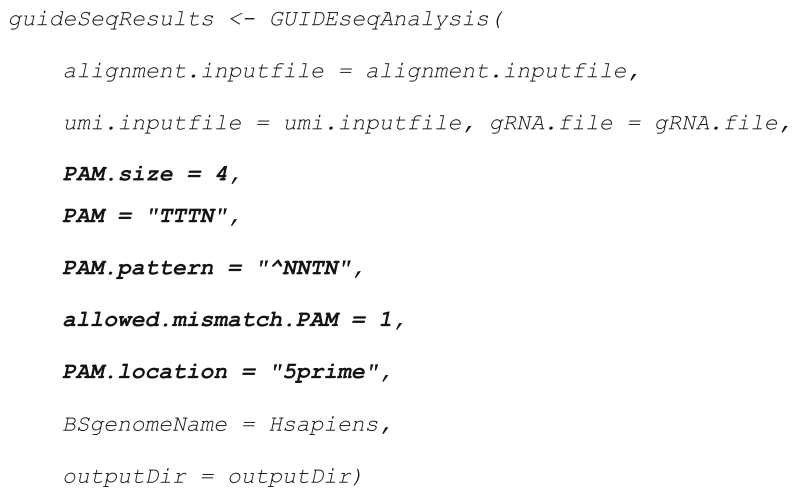

Another advantage of our GUIDEseq package is the ability/flexibility to plug in additional annotation packages within Bioconductor. Although we only present examples for the analysis of human genome datasets, BSgenomeName can be set to analyze data from other species, such as the Mmusculus package for mouse (BSgenome.Mmusculus.UCSC.mm10), the Rnorvegicus package for rat (BSgenome.Rnorvegicus.UCSC.rn6), the Scerevisiae package for yeast (BSgenome.Scerevisiae.UCSC.sacCer3), the Celegans package for C. elegans (BSgenome.Celegans.UCSC.ce11), and the Dmelanogaster package for D. melanogaster (BSgenome.Dmelanogaster.UCSC.dm6). For a list of available species-specific BSgenomes, please search for keyword "BSgenome" at https://bioconductor.org/packages/3.3/BiocViews.html\#__AnnotationData. For genomes not available as Bioconductor packages, users or the core team can create one using the utility detailed at http://www.bioconductor.org/packages/release/bioc/ vignettes/BSgenome/inst/doc/BSgenomeForge.pdf.

\section{Example 4. Annotate off-targets}

With parameters $t x d b$ and organAnn set to an organismspecific transcript object and gene ID mapping object, offtarget sites are annotated if they overlap with gene bodies and if they fall within an exon. Here is an example for SpCas9 GUIDE-seq data processing that annotates identified potential off-target sites with features from the human genome.

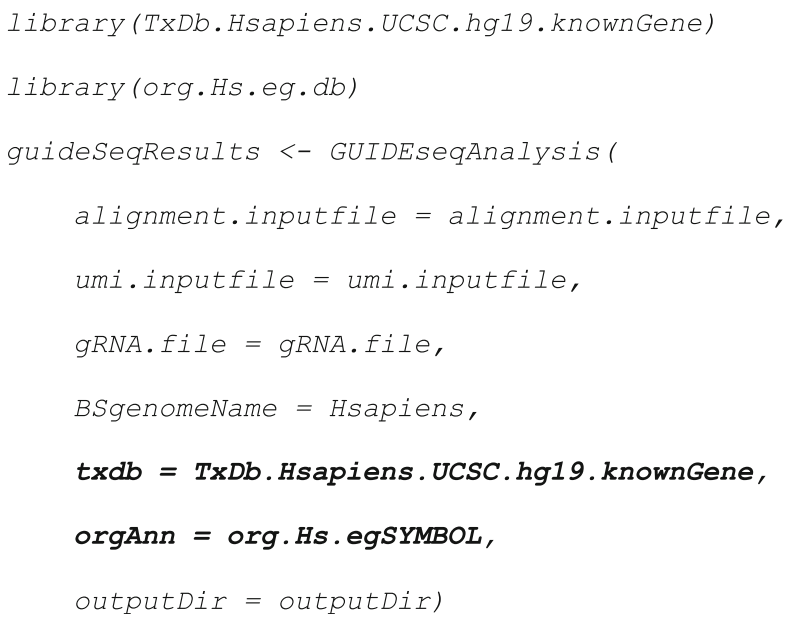

To annotate off-targets in other genomes, set $t x d b$ and orgAnn accordingly. For example, set $t x d b$ to TxDb.Mmus culus.UCSC.mm10.knownGene and orgAnno to org.M m.eg.db for mouse, txdb to TxDb.Rnorvegicus.UCSC.rn6. refGene and orgAnno to org.Rn.eg.db for rat, txdb to TxDb.Dmelanogaster.UCSC.dm6.ensGene and orgAnno to org.Dm.eg.db for $D$. melanogaster, and txdb to TxDb.Celegans.UCSC.ce11.ensGene and orgAnno to org.Ce.eg.db for C. elegans. For a list of existing $\mathrm{TxDb}$ and gene ID mapping packages search for keywords "Txdb" and "OrgDb" at http://www.bioconductor.org/packages/ release/BiocViews.html\#_AnnotationData. Please refer to GenomicFeatures package for creation of additional transcript packages.

Example 5. Merge off-targets from multiple experiments to facilitate comparisons among different nuclease configurations or variants

When evaluating novel nuclease treatment conditions or different Cas9 variants, it is common practice to 
include off-target analysis of standard platforms as controls. To aid in comparisons between different nucleases, off-targets identified by GUIDE-seq can be easily merged using the combineOfftargets function. Here is the example code to merge three experiments and generate a Venn diagram to depict the off-target overlaps among experiments (Fig. 4).

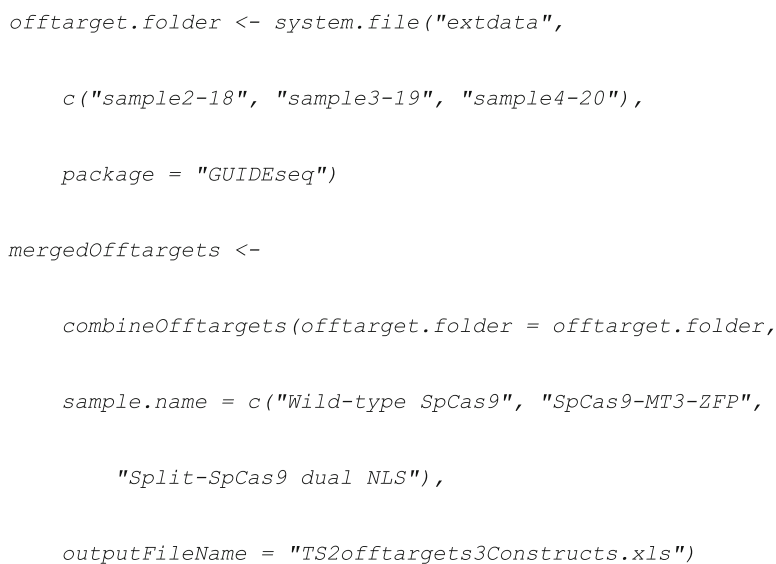

If desired combineOfftargets can be used to remove off-targets common among different gRNAs by setting remove.common to TRUE. Furthermore, if a control sample without nuclease is available, peaks present in the control sample can be removed from the gRNA samples by setting the control.sample.name.

\section{Conclusions}

GUIDEseq provides a flexible analysis platform for the identification and annotation of nuclease-based offtarget cleavage sites that are tagged through the GUIDE-seq methodology developed by the Joung

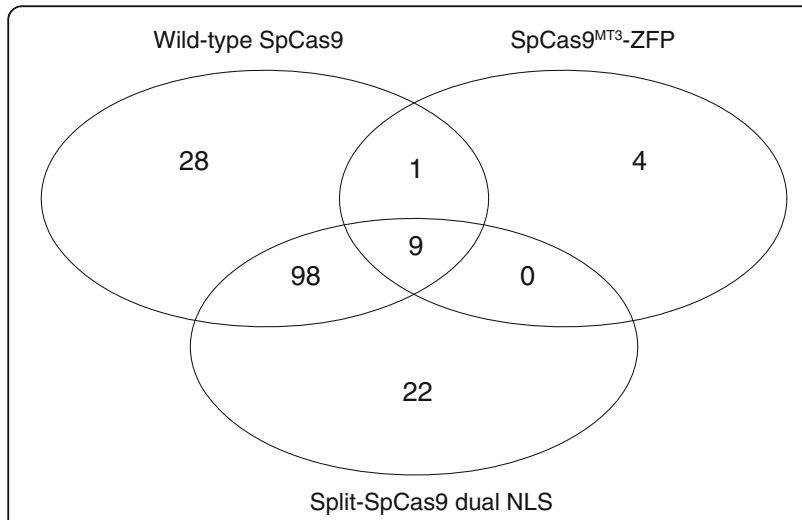

Fig. 4 Venn Diagram generated using combineOfftargets to depict the overlaps of off-target sites between three different nuclease variants. Example of the output from the combineOfftargets function (Example 6) comparing the overlap in GUIDE-seq identified offtarget sites for wild-type Cas9, Split-Cas9 (dual NLS) [51], and the highly specific SpCas9 ${ }^{\text {MT3 }}$-ZFP [25] programmed with a sgRNA recognizing VEGFA site 2 (TS2) [11] laboratory [19]. Harnessing the diverse software resources and databases available within Bioconductor $[45,46,48,50]$, GUIDEseq provides a streamlined environment for the identification of off-target sites in a wide variety of species. In comparison to the recently released guideseq analysis pipeline in python [40], our package provides a rich parameter set that allows users to easily modify the processing of GUIDE-seq data to adapt to a variety of different types of nucleases by accommodating different target sequence characteristics, such as gRNA length, canonical PAM sequence composition and position of the PAM relative to the protospacer. In addition, GUIDEseq allows the definition of different filtering and peak calling criteria, as well as different target site complementarity thresholds for both the guide sequence and PAM element for the capture of potential off-target sequences that are associated with GUIDE-seq peaks. This flexibility, which is absent in the python analysis pipeline [40], allows the differential tuning of these features for the more liberal capture of potential offtarget sites for subsequent validation.

Furthermore, our GUIDEseq package has a number of additional distinct features. It can output an off-target cleavage prediction score for each site based on the complementarity to the input target sequence using activity models generated from a variety of experimental datasets $[5,12]$. Deviations in the off-target cleavage rates from the predicted score may identify sites where biological factors are impacting nuclease cleavage rates, which could inform subsequent iterations of these activity models. Our GUIDEseq package also permits the utilization of different annotation packages such as BSgenome and $T x D b$ to define putative off-target sites that overlap features of interest within a genome. This information can be used to prioritize the validation of identified potential off-target sites. In addition, our package allows a comparative analysis of nuclease precision and visualization of identified off-target sites from different experiments in a Venn diagram. This feature may be particularly valuable when different nuclease treatment conditions or nuclease platforms are being compared to define the most promising nuclease framework to pursue for future studies.

\section{User information}

A step-by-step user guide with working code snippets for the GUIDEseq analysis package is available at http:// bioconductor.org/packages/release/bioc/vignettes/GUID Eseq/inst/doc/GUIDEseq.pdf. Detailed parameter definition, default setting and usage are available at https:// bioconductor.org/packages/release/bioc/manuals/GUIDE $\mathrm{seq} / \mathrm{man} /$ GUIDEseq.pdf. GUIDEseq depends on $\mathrm{R}$ version 3.3.0 or later. 


\section{Additional files}

Additional file 1: Supplemental Methods for GUIDE-seq data preprocessing. (PDF $166 \mathrm{~kb}$ )

Additional file 2: Installation and Usage of GUIDEseq for novice $R$ users. (PDF $224 \mathrm{~kb}$ )

Additional file 3: Table S1. An example output of GUIDEseq analysis. (XLSX $72 \mathrm{~kb}$ )

Additional file 4: Table S2. Common and unique off-targets identified by GUIDEseq and published in Tsai 2015 [19]. (XLSX 32 kb)

\section{Abbreviations}

CRISPR: Clustered regularly interspaced short palindromic repeats; DSB: Doublestrand break; GUIDE-seq: Genome-wide unbiased identification of DSBs enabled by sequencing; PAM: Protospacer adjacent motif

\section{Acknowledgements}

The authors would like to thank Shengdar Tsai \& J. Keith Joung for sharing sequencing data of HEK239_site4 for the comparative analysis.

\section{Funding}

This work was supported by NIH grant R01Al117839 \& R01HL093766 to S.A.W.

\section{Availability of data and materials}

GUIDEseg is an open source software package under the GNU General Public License v2.0 and has been contributed to the Bioconductor Project. The software, source code and documentation are available for download from http://www.bioconductor.org/packages/release/bioc/html/ GUIDEseq.html or installed from R by typing source("http://bioconductor.org/ biocLite.R") and biocLite("GUIDEseq"). The datasets analyzed in this study were generously supplied by the Joung laboratory or were generated in house.

\section{Authors' contributions}

LJZ and SAW conceived and designed the computational method. LJZ, ML and HP developed the software package. LJZ and AG analyzed the data. LJZ and SAW wrote the paper. All authors read and approved the final manuscript.

\section{Competing interests}

The authors have filed patent applications related to genome engineering technologies. S.A.W. is a consultant for Editas Medicine.

\section{Consent for publication}

Not applicable.

\section{Ethics approval and consent to participate}

Not applicable.

\section{Publisher's Note}

Springer Nature remains neutral with regard to jurisdictional claims in published maps and institutional affiliations.

\footnotetext{
Author details

${ }^{1}$ Department of Molecular, Cell and Cancer Biology, University of Massachusetts Medical School, Worcester, MA, USA. ²Program in Bioinformatics and Integrative Biology, University of Massachusetts Medical School, Worcester, MA, USA. ${ }^{3}$ Department of Molecular Medicine, University of Massachusetts Medical School, Worcester, MA, USA. ${ }^{4}$ Genentech, San Francisco, CA, USA. ${ }^{5}$ Program in Computational Biology, Fred Hutchinson Cancer Research Center, Seattle, WA 98109-1024, USA. ${ }^{6}$ Department of Biochemistry and Molecular Pharmacology, University of Massachusetts Medical School, Worcester, MA, USA.
}

Received: 27 March 2017 Accepted: 1 May 2017

Published online: 15 May 2017

\section{References}

1. Sontheimer EJ, Barrangou R. The bacterial origins of the CRISPR genomeediting revolution. Hum Gene Ther. 2015;26(7):413-24.

2. Doudna JA, Charpentier E. Genome editing. The new frontier of genome engineering with CRISPR-Cas9. Science (New York, NY). 2014;346(6213): 1258096.

3. Sander JD, Joung JK. CRISPR-Cas systems for editing, regulating and targeting genomes. Nat Biotechnol. 2014;32(4):347-55.

4. Thyme SB, Akhmetova L, Montague TG, Valen E, Schier AF. Internal guide RNA interactions interfere with Cas9-mediated cleavage. Nat Commun. 2016;7:11750

5. Doench JG, Fusi N, Sullender M, Hegde M, Vaimberg EW, Donovan KF, Smith I, Tothova Z, Wilen C, Orchard R, et al. Optimized sgRNA design to maximize activity and minimize off-target effects of CRISPR-Cas9. Nat Biotechnol. 2016;34(2):184-91.

6. Ledford H. CRISPR, the disruptor. Nature. 2015;522:20-4.

7. Cox DBT, Platt RJ, Zhang F. Therapeutic genome editing: prospects and challenges. Nat Med. 2015;21(2):121-31.

8. Tycko J, Myer VE, Hsu PD. Methods for Optimizing CRISPR-Cas9 Genome Editing Specificity. Mol Cell. 2016;63(3):355-70.

9. Jinek M, Chylinski K, Fonfara I, Hauer M, Doudna JA, Charpentier E. A programmable dual-RNA-guided DNA endonuclease in adaptive bacterial immunity. Science. 2012;337(6096):816-21.

10. Cong L, Ran FA, Cox D, Lin S, Barretto R, Habib N, Hsu PD, Wu X, Jiang W, Marraffini LA, et al. Multiplex genome engineering using CRISPR/Cas systems. Science. 2013;339(6121):819-23.

11. Fu Y, Foden JA, Khayter C, Maeder ML, Reyon D, Joung JK, Sander JD. Highfrequency off-target mutagenesis induced by CRISPR-Cas nucleases in human cells. Nat Biotechnol. 2013;31(9):822-6.

12. Hsu PD, Scott DA, Weinstein JA, Ran FA, Konermann S, Agarwala V, Li Y, Fine EJ, Wu X, Shalem O, et al. DNA targeting specificity of RNA-guided Cas9 nucleases. Nat Biotechnol. 2013;31(9):827-32.

13. Cho SW, Kim S, Kim Y, Kweon J, Kim HS, Bae S, Kim JS. Analysis of off-target effects of CRISPR/Cas-derived RNA-guided endonucleases and nickases. Genome Res. 2014;24(1):132-41.

14. Pattanayak V, Lin S, Guilinger JP, Ma E, Doudna JA, Liu DR. High-throughput profiling of off-target DNA cleavage reveals RNA-programmed Cas9 nuclease specificity. Nat Biotechnol. 2013;31(9):839-43.

15. Lin Y, Cradick TJ, Brown MT, Deshmukh H, Ranjan P, Sarode N, Wile BM, Vertino PM, Stewart FJ, Bao G. CRISPR/Cas9 systems have off-target activity with insertions or deletions between target DNA and guide RNA sequences. Nucleic Acids Res. 2014;42(11):7473-85.

16. Mali P, Aach J, Stranges PB, Esvelt KM, Moosburner M, Kosuri S, Yang L, Church GM. CAS9 transcriptional activators for target specificity screening and paired nickases for cooperative genome engineering. Nat Biotechnol. 2013;31(9):833-8.

17. Wu X, Scott DA, Kriz AJ, Chiu AC, Hsu PD, Dadon DB, Cheng AW, Trevino $A E$, Konermann S, Chen S, et al. Genome-wide binding of the CRISPR endonuclease Cas9 in mammalian cells. Nat Biotechnol. 2014;32(7):670-6.

18. Kuscu C, Arslan S, Singh R, Thorpe J, Adli M. Genome-wide analysis reveals characteristics of off-target sites bound by the Cas9 endonuclease. Nat Biotechnol. 2014:32(7):677-83.

19. Tsai SQ, Zheng Z, Nguyen NT, Liebers M, Topkar W, Thapar V, Wyvekens N, Khayter C, lafrate AJ, Le LP, et al. GUIDE-seq enables genome-wide profiling of off-target cleavage by CRISPR-Cas nucleases. Nat Biotechnol. 2015:33(2):187-97.

20. Kim D, Bae S, Park J, Kim E, Kim S, Yu HR, Hwang J, Kim J-I, Kim J-S. Digenome-seq: genome-wide profiling of CRISPR-Cas9 off-target effects in human cells. Nat Methods. 2015;12(3):237-43.

21. Ran FA, Cong L, Yan WX, Scott DA, Gootenberg JS, Kriz AJ, Zetsche B, Shalem O, Wu X, Makarova KS, et al. In vivo genome editing using Staphylococcus aureus Cas9. Nature. 2015;520(7546):186-91.

22. Shen B, Zhang W, Zhang J, Zhou J, Wang J, Chen L, Wang L, Hodgkins A, lyer $V$, Huang $X$, et al. Efficient genome modification by CRISPR-Cas9 nickase with minimal off-target effects. Nat Methods. 2014;11(4):399-402.

23. Tsai SQ, Wyvekens N, Khayter C, Foden JA, Thapar V, Reyon D, Goodwin MJ, Aryee MJ, Joung JK. Dimeric CRISPR RNA-guided Fokl nucleases for highly specific genome editing. Nat Biotechnol. 2014;32(6):569-76. 
24. Guilinger JP, Thompson DB, Liu DR. Fusion of catalytically inactive Cas9 to Fokl nuclease improves the specificity of genome modification. Nat Biotechnol. 2014;32(6):577-82.

25. Bolukbasi MF, Gupta A, Oikemus S, Derr AG, Garber M, Brodsky MH, Zhu LJ, Wolfe SA. DNA-binding-domain fusions enhance the targeting range and precision of Cas9. Nat Methods. 2015;12(12):1150-6.

26. Slaymaker IM, Gao L, Zetsche B, Scott DA, Yan WX, Zhang F. Rationally engineered Cas9 nucleases with improved specificity. Science (New York, NY). 2016;351(6268):84-8.

27. Kleinstiver BP, Pattanayak V, Prew MS, Tsai SQ, Nguyen NT, Zheng Z, Joung JK. High-fidelity CRISPR-Cas9 nucleases with no detectable genome-wide off-target effects. Nature. 2016;529(7587):490-5.

28. Kleinstiver BP, Prew MS, Tsai SQ, Topkar W, Nguyen NT, Zheng Z, Gonzales APW, Li Z, Peterson RT, Yeh J-RJ, et al. Engineered CRISPR-Cas9 nucleases with altered PAM specificities. Nature. 2015;523(7561):481-5.

29. Kleinstiver BP, Prew MS, Tsai SQ, Nguyen NT, Topkar W, Zheng Z, Joung JK. Broadening the targeting range of Staphylococcus aureus CRISPR-Cas9 by modifying PAM recognition. Nat Biotechnol. 2015;33(12):1293-8.

30. Lee CM, Cradick TJ, Bao G. The Neisseria meningitidis CRISPR-Cas9 System Enables Specific Genome Editing in Mammalian Cells. Mol Ther. 2016;24(3):645-54.

31. Kim D, Kim J, Hur JK, Been KW, Yoon SH, Kim JS. Genome-wide analysis reveals specificities of Cpf1 endonucleases in human cells. Nat Biotechnol. 2016;34(8):863-8.

32. Kleinstiver BP, Tsai SQ, Prew MS, Nguyen NT, Welch MM, Lopez JM, McCaw ZR, Aryee MJ, Joung JK. Genome-wide specificities of CRISPR-Cas Cpf1 nucleases in human cells. Nat. Biotechnol. 2016;34:869-74.

33. Frock RL, Hu J, Meyers RM, Ho YJ, Kii E, Alt FW. Genome-wide detection of DNA double-stranded breaks induced by engineered nucleases. Nat Biotechnol. 2015;33(2):179-86.

34. Lupiáñez DG, Kraft K, Heinrich V, Krawitz P, Brancati F, Klopocki E, Horn D, Kayserili H, Opitz JM, Laxova R, et al. Disruptions of topological chromatin domains cause pathogenic rewiring of gene-enhancer interactions. Cell. 2015;161(5):1012-25.

35. Torres R, Martin MC, Garcia A, Cigudosa JC, Ramirez JC, Rodriguez-Perales S. Engineering human tumour-associated chromosomal translocations with the RNA-guided CRISPR-Cas9 system. Nat Commun. 2014;5:3964.

36. Choi PS, Meyerson M. Targeted genomic rearrangements using CRISPR/Cas technology. Nat Commun. 2014;5:3728.

37. Ghezraoui H, Piganeau M, Renouf B, Renaud J-B, Sallmyr A, Ruis B, Oh S, Tomkinson AE, Hendrickson EA, Giovannangeli $C$, et al. Chromosomal translocations in human cells are generated by canonical nonhomologous end-joining. Mol Cell. 2014;55(6):829-42.

38. Wang $X$, Wang $Y$, Wu X, Wang J, Wang Y, Qiu Z, Chang T, Huang $H$, Lin R-J, Yee J-K. Unbiased detection of off-target cleavage by CRISPRCas9 and TALENs using integrase-defective lentiviral vectors. Nat Biotechnol. 2015;33(2):175-8.

39. Yin $H$, Song CQ, Dorkin JR, Zhu LJ, Li Y, Wu Q, Park A, Yang J, Suresh S, Bizhanova A, et al. Therapeutic genome editing by combined viral and nonviral delivery of CRISPR system components in vivo. Nat Biotechnol. 2016; 34(3):328-33.

40. Tsai SQ, Topkar W, Joung JK, Aryee MJ. Open-source guideseq software for analysis of GUIDE-seq data. Nat Biotechnol. 2016;34(5):483.

41. Hou Z, Zhang Y, Propson NE, Howden SE, Chu LF, Sontheimer EJ, Thomson JA. Efficient genome engineering in human pluripotent stem cells using Cas9 from Neisseria meningitidis. Proc Natl Acad Sci U S A. 2013;110(39): 15644-9.

42. Zetsche B, Gootenberg JS, Abudayyeh OO, Slaymaker IM, Makarova KS, Essletzbichler P, Volz SE, Joung J, van der Oost J, Regev A, et al. Cpf1 Is a Single RNA-Guided Endonuclease of a Class 2 CRISPR-Cas System. Cell. 2015; 163(3):759-71. doi:10.1016/j.cell.2015.09.038.

43. Ihaka R, Gentleman R. R: A language for data analysis and graphics. J Comput Graph Stat. 1996:5:299-314.

44. Gentleman RC, Carey VJ, Bates DM, Bolstad B, Dettling M, Dudoit S, Ellis B, Gautier L, Ge Y, Gentry J, et al. Bioconductor: open software development for computational biology and bioinformatics. Genome Biol. 2004;5(10):R80.

45. Lawrence M, Huber W, Pagès $H$, Aboyoun P, Carlson M, Gentleman R, Morgan MT, Carey VJ. Software for computing and annotating genomic ranges. PLoS Comput Biol. 2013;9(8):e1003118.
46. Zhu LJ, Gazin C, Lawson ND, Pagès H, Lin SM, Lapointe DS, Green MR. ChIPpeakAnno: a bioconductor package to annotate ChIP-seq and ChIPchip data. BMC Bioinf. 2010;11(1):237.

47. Zhu LJ. Integrative analysis of ChIP-chip and ChIP-seg dataset. Methods Mol Biol. 2013;1067:105-24

48. Zhu LJ, Holmes BR, Aronin N, Brodsky MH. CRISPRseek: a bioconductor package to identify target-specific guide RNAs for CRISPR-Cas9 genomeediting systems. PLoS ONE. 2014;9(9):e108424.

49. Zhu LJ. Overview of guide RNA design tools for CRISPR-Cas9 genome editing technology. Frontiers in Biology. 2015;10(4):289-96. http://link. springer.com/article/10.1007\%2Fs11515-015-1366-y.

50. Ritchie ME, Phipson B, Wu D, Hu Y, Law CW, Shi W, Smyth GK. limma powers differential expression analyses for RNA-sequencing and microarray studies. Nucleic Acids Res. 2015;43(7):e47.

51 Zetsche B, Volz SE, Zhang F. A split-Cas9 architecture for inducible genome editing and transcription modulation. Nat Biotechnol. 2015;33(2):139-42.

\section{Submit your next manuscript to BioMed Central and we will help you at every step:}

- We accept pre-submission inquiries

- Our selector tool helps you to find the most relevant journal

- We provide round the clock customer support

- Convenient online submission

- Thorough peer review

- Inclusion in PubMed and all major indexing services

- Maximum visibility for your research

Submit your manuscript at www.biomedcentral.com/submit
Biomed Central 